Document downloaded from:

http://hdl.handle.net/10251/54641

This paper must be cited as:

Gonzalez Iglesias, D.; Soto Pacheco, P.; Anza Hormigo, S.; Gimeno Martinez, B.; Boria Esbert, VE.; Vicente Quiles, CP.; Gil Raga, J. (2012). Multipactor susceptibility charts for ridge and multiridge waveguides. IEEE Transactions on Electron Devices. 59(12):36013607. doi:10.1109/TED.2012.2215611.

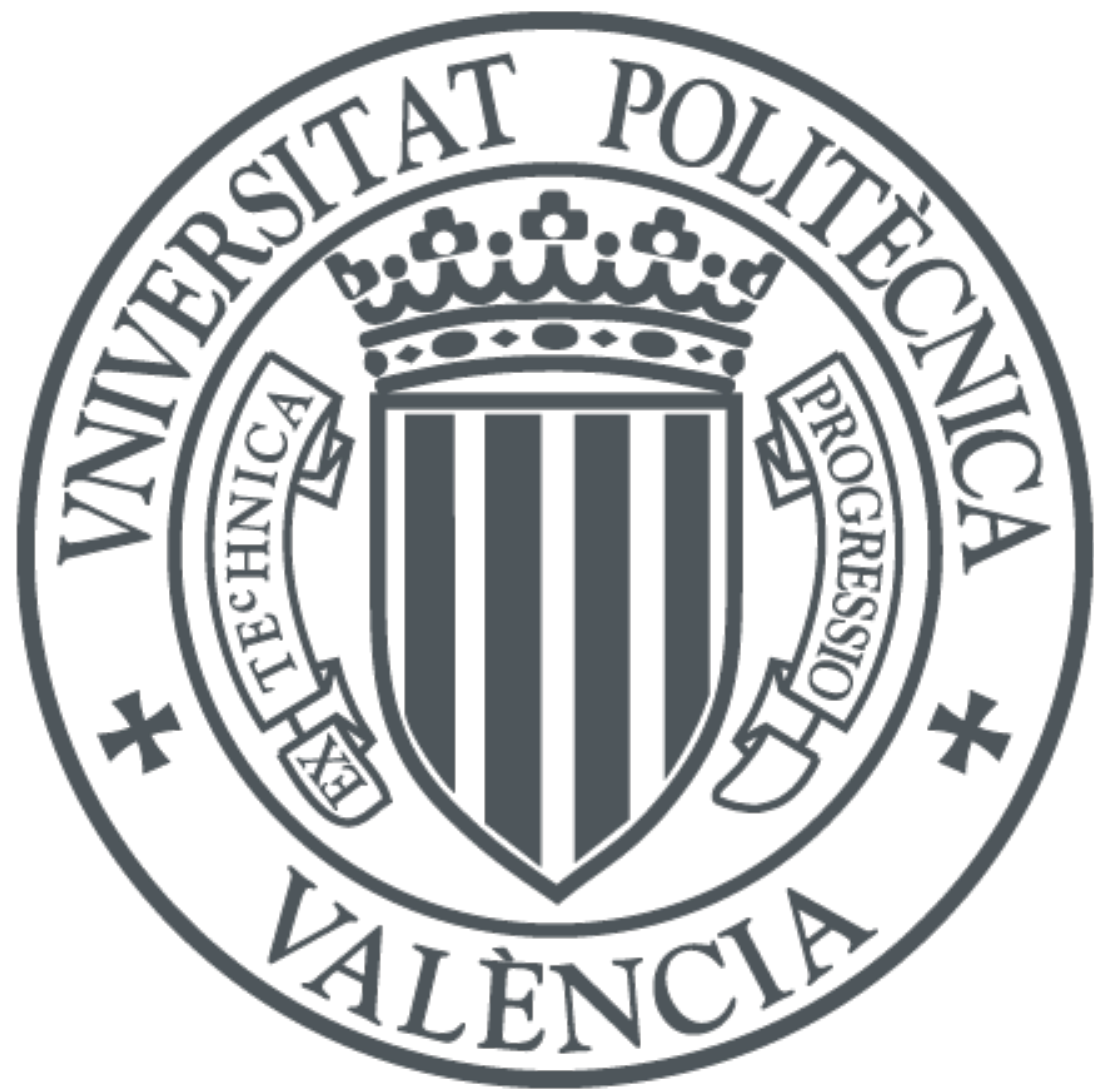

The final publication is available at

http://dx.doi.org/10.1109/TED.2012.2215611

Copyright Institute of Electrical and Electronics Engineers (IEEE)

Additional Information

"(C) 2012 IEEE. Personal use of this material is permitted. Permission from IEEE must be obtained for all other uses, in any current or future media, including reprinting/republishing this material for advertising or promotional purposes, creating new collective works, for resale or redistribution to servers or lists, or reuse of any copyrighted component of this work in other works." 


\title{
Multipactor Susceptibility Charts for Ridge and Multi-Ridge Waveguides
}

\author{
D. González-Iglesias, P. Soto, Member, IEEE, S. Anza, B. Gimeno, Member, IEEE, \\ V. E. Boria, Senior Member, IEEE, C. Vicente, Member, IEEE, J. Gil, Member, IEEE
}

\begin{abstract}
The aim of this letter is to study the multipactor RF breakdown voltage in several ridge and multi-ridge waveguide configurations. Firstly, multipactor susceptibility charts for several types of ridged waveguides have been computed using the commercial software FEST3D. Next, these charts have been used to predict multipactor threshold values for a band-pass filter and a quasi low-pass filter both containing ridge waveguide sections. Furthermore, multipactor simulations using FEST3D are carried out to calculate the multipactor threshold of the aforementioned structures. A good agreement between predictions and simulations has been found for both filter examples.
\end{abstract}

Index Terms-Ridge waveguide, Multi-ridge waveguide, Multipactor effect, RF breakdown.

\section{INTRODUCTION}

Multipactor is a vacuum discharge phenomenon that takes place on devices operating under high-power radio frequency (RF) electromagnetic fields [1]. It can appear in a wide variety of scenarios, such as satellite communication devices, klystrons and accelerator structures [2]-[3]. The multipactor effect occurs when an RF electric field accelerates free electrons inside the device impacting on the metallic walls of the device. When electron impact energy is such that the secondary emission yield coefficient (SEY), $\delta$ of the material is higher than unity, new secondary electrons are released [4]. Under certain resonant conditions, new secondaries get synchronized with the RF electric field producing an exponential growth of the electron population. Once the number of electrons becomes very high, an electrical current is established between the walls of the component. This RF discharge can produce several negative effects that degrade the device performance: increase of signal noise and reflected power, heating up of the device walls, outgassing, detuning of resonant cavities, vacuum window failure and even the total destruction of the component.

Multipactor has been deeply studied for many different kinds of geometries such as parallel-plate [5]-[9], coaxial [10][13], rectangular [14]-[15], microstrip [16], circular [17]-[18], and elliptical [19] waveguides. Despite all these past efforts,

D. González-Iglesias and B. Gimeno are with the Departamento de Física Aplicada, Instituto de Ciencia de Materiales, Universidad de Valencia, 46100Burjasot(Valencia), Spain.

P. Soto and V.E. Boria are with the Departamento de Comunicaciones, Instituto de Telecomunicaciones y Aplicaciones Multimedia, Universidad Politécnica de Valencia, 46022-Valencia, Spain.

S. Anza, C. Vicente and J. Gil are with Aurora Software and Testing, S.L., Business Development Building, Universidad Politécnica de Valencia, 46022Valencia, Spain. and to the authors' knowledge, multipactor in ridge and multiridge waveguides has not been studied yet.

Single- and multi-ridged waveguides have found many applications in microwave and millimeter-wave devices. Among their main advantages we find that they include large singlemode broad-band operation, large dominant cutoff wavelength, and low impedance characteristics. They are widely used for both high-power and low-power applications such as band-pass and quasi low-pass filters [20]. These filters are very appropriate candidates for some space and terrestrial communications applications due to their compact size and good stop-band performance. For instance, they can be used as preselector filters before the input multiplexer, or as harmonic suppression filters after RF transmitters or amplifiers, particulary as on board satellite components.

In this work, we have computed multipactor susceptibility charts for several ridge and multi-ridge waveguide configurations. First, section II describes the procedure carried out to perform the requested multipactor simulations in order to generate the susceptibility charts. In section III, ridge, double-ridge and multi-ridge waveguide multipactor charts are presented for both symmetrical and asymmetrical configurations. These results are used in section IV for predicting the multipactor threshold values of an evanescent-mode filter, and of a waffle-iron filter, both containing ridge-waveguide sections. In addition, and with the aim of verifying the derived susceptibility charts, a complete FEST3D multipactor analysis has been performed for both filter structures. Finally, in section $\mathrm{V}$ some relevant conclusions are outlined.

\section{Multipactor Analysis Methodology}

The commercial software FEST3D [21] has been used to perform multipactor simulations within the analyzed ridged waveguides. This code allows 3-D tracking of a set of electrons immersed into a region under RF electric and magnetic fields. An electromagnetic solver based on full-wave modal techniques [22] and microwave network theory calculates these fields with high accuracy and efficiency, even for complex passive microwave components. The structures are excited from the input port with its fundamental mode. Differential equations of electrons motion are numerically solved using a Leap-Frog method, as described in [23]. The interactions of the electrons with the metal boundaries are modeled to consider electron elastic reflection, absorption or creation of secondaries after each primary electron impact. The SEY is modeled using a modified version of the Vaughan's formula [14]. All 

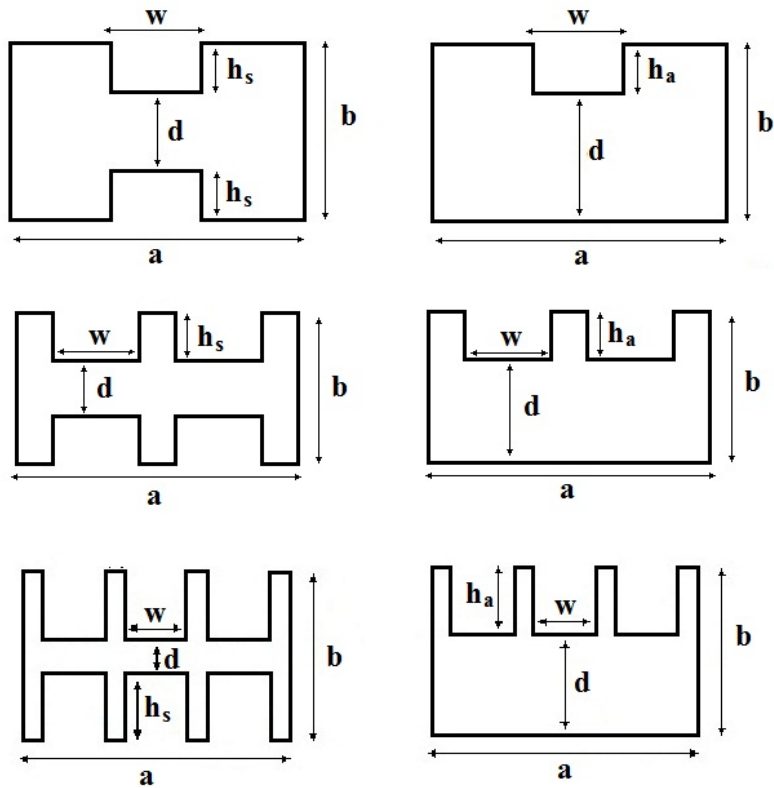

Fig. 1. Symmetrical structures on the left column and asymmetrical structures on the right column. From top to bottom: ridge, double-ridge and multi-ridge waveguides.

simulated structures are silver-plated. The SEY parameters for silver, which are described in [6], are: the first cross-over, $W_{1}=30 \mathrm{eV}$; the maximum SEY coefficient, $\delta_{\max }=2.22$; and the impact kinetic energy for $\delta_{\max }, W_{\max }=165 \mathrm{eV}$. The secondary electron velocities have been computed using a 4 $\mathrm{eV}$ mean and $2 \mathrm{eV}$ standard deviation Maxwellian distribution.

\section{MultipaCtor Susceptibility CHARTS}

Ridge, double-ridge and multi-ridge waveguides (see Fig. 1) with housing dimensions of a standard WR75 rectangular ( $a=19.05 \mathrm{~mm}, b=9.525 \mathrm{~mm}$ ) have been analyzed. Symmetrical and asymmetrical configurations have been considered. In susceptibility charts, the RF multipactor voltage threshold is depicted as a function of the frequency gap $f \times d$.

Multipactor discharge is always expected to occur in the narrowest waveguide zone, where electric field becomes higher. According to this, the existing gap between metal ridges is the critical zone for the appearance of a multipactor discharge. Thus, an equivalent voltage is numerically computed using the following expression:

$$
V_{e q}=\left|\int_{0}^{d} \vec{E} \cdot \overrightarrow{d l}\right|
$$

where $\vec{E}$ is the RF electric field of the fundamental mode in the ridge gap, and $\overrightarrow{d l}$ is the differential vector parallel to the transversal component of the electric field. It can be checked numerically that for double-ridge waveguides the equivalent voltage is the same at both symmetrical ridges, whereas for multi-ridge waveguides (with an odd number of ridges) the voltage is higher at the central one. In Figs. 2 and Fig. 3 the electric field lines are depicted for the different waveguide geometries.
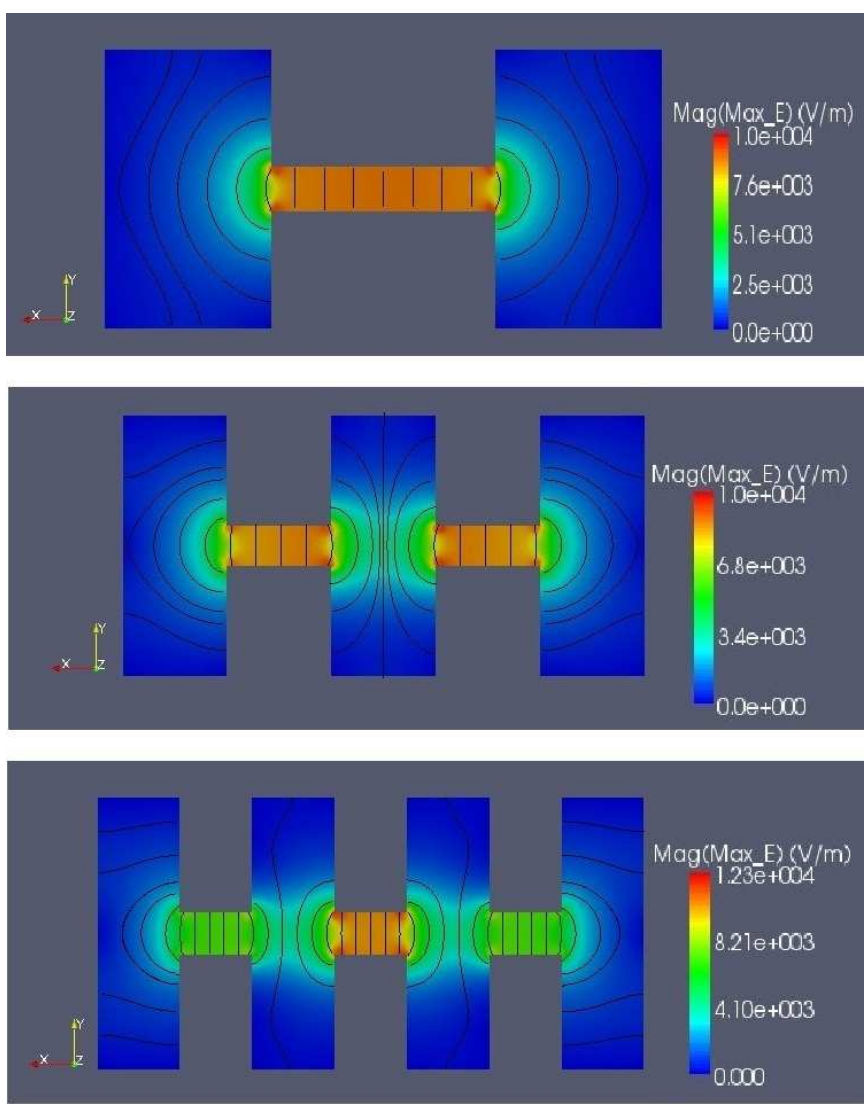

Fig. 2. Electric field lines for symmetrical configurations. From top to bottom: ridge, double-ridge and multi-ridge waveguides.

Fig. 4 shows the voltage threshold as a function of the waveguide length for both symmetrical and asymmetrical single-ridge waveguides. Multipactor simulations show that multipactor threshold strongly depends on the waveguide length value, i.e. $l / \lambda_{g}$ in Fig. 4. In fact, the voltage threshold raises up for short waveguide lengths as a result of the axial drift that allows electrons leaving the ridge gap. Thus, in order to counteract the lost electrons a higher rate of secondaries generation is needed, which is obtained by means of an increase of RF power. Similar results were obtained for the different multi-ridge topologies. In the next multipactor susceptibility charts, a waveguide length in the flat zone of the graphic has been always considered (i.e. $l / \lambda_{g}>0.1$ ).

Two different kinds of charts are presented. First, RF voltage threshold for several ridge widths is depicted in Figs. 5 and 6 for symmetrical and asymmetrical configurations, respectively. As it can be shown, there are slight variations on voltage threshold with the ridge width for each figure. However, it can be observed that the narrower the ridge the higher the voltage threshold. An explanation of this phenomenon is that lower $w / a$ values allow electrons to escape from the gap region, so an extra voltage is needed in order to compensate this phenomenon. Similar multipactor behavior is found for waveguide irises [24]-[26], where the power threshold increases when the $h / l$ ratio is higher ( $h$ is the gap height and $l$ is the iris length). Two different mechanisms for electron loss were proposed for an iris scenario. On the one 


\section{Page 3 of 8}
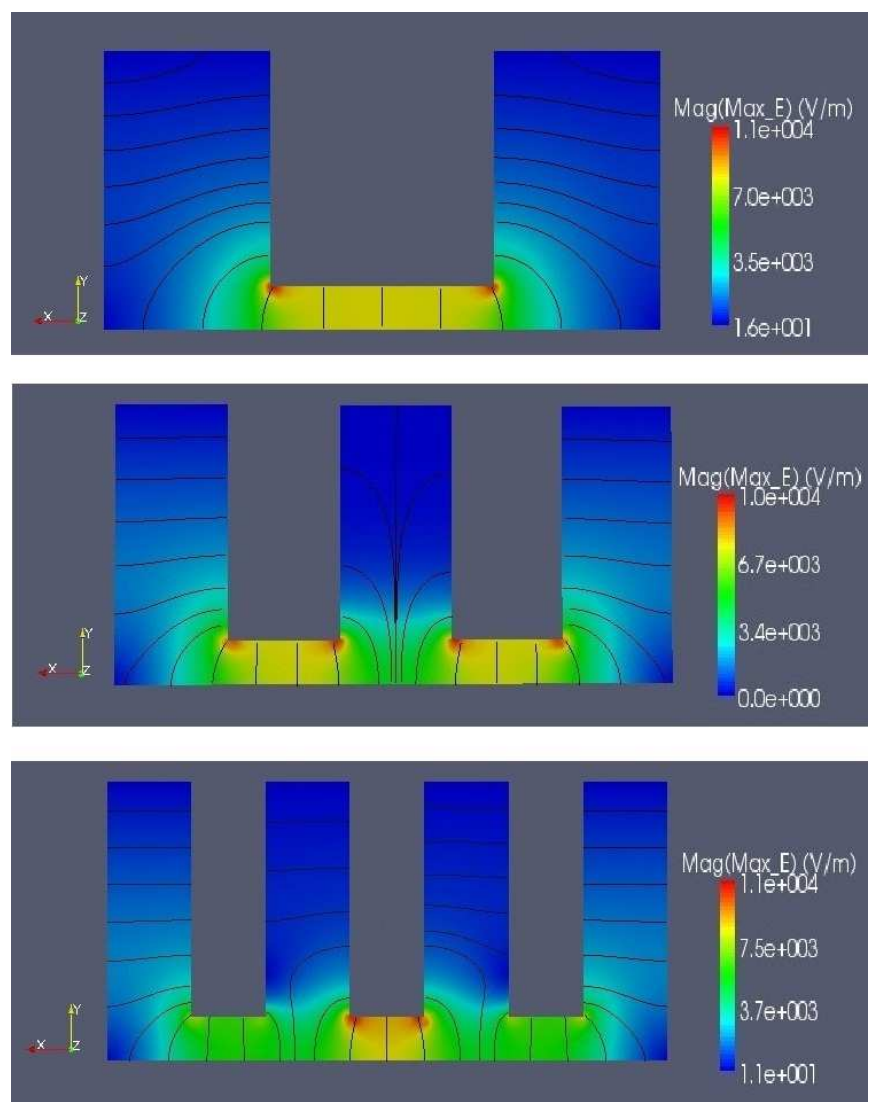

Fig. 3. Electric field lines for asymmetrical configurations. From top to bottom: ridge, double-ridge and multi-ridge waveguides.

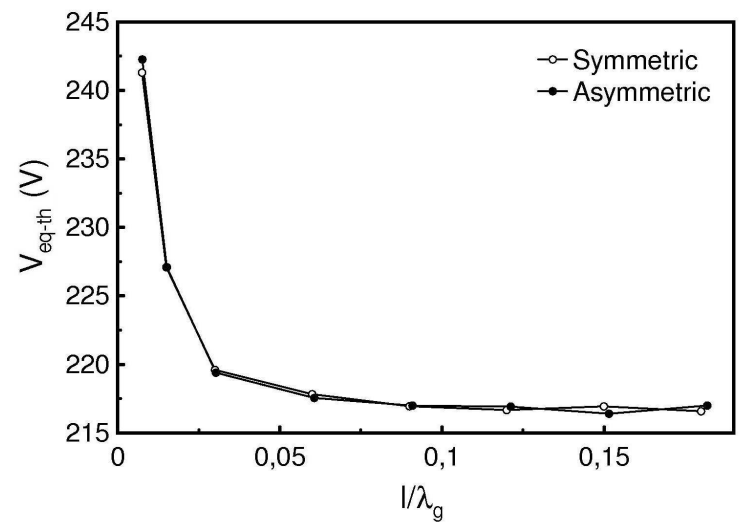

Fig. 4. Multipactor voltage threshold as a function of the normalized waveguide length for symmetrical and asymmetrical single-ridge waveguides. Gap is $d=0.5 \mathrm{~mm}(d / b=0.052), b / a=2$ and $w / a=0.4$. RF frequency is $5 \mathrm{GHz}$ and $\lambda_{g}$ is the wavelength in the guide.

hand, fringing electric field accelerates electrons out of the iris. On the other hand, a random drift due to the axial component of the initial velocity of the secondary emitted electrons may push many electrons away from the gap region. In our ridge waveguide case, both effects are supposed to act but in the transverse plane to the wave propagation, specifically parallel to the ridge width of value $w$.

For a better understanding of the fringing phenomenon in


Fig. 5. Multipactor voltage threshold for several ridge widths in symmetrical configurations. From top to bottom: ridge, double-ridge and multi-ridge waveguides. Gap length is $d=0.5 \mathrm{~mm}, d / b=0.052$ and $b / a=2$.

ridged waveguides, an study of the voltage threshold dependance with the ratio of ridge width to gap, $w / d$, has been performed. It covers a wider range of $w / d$ values than the previous $w / a$ analysis. Results are presented in Fig. 7 for a symmetrical single ridge waveguide but similar behavior can be found for asymmetrical and/or multi-ridge waveguides. It is observed than fringing effect raises up voltage threshold as $w / d$ ratio reduces. This behavior becomes more important when $w / d$ is close to unity or lower. On the other hand, for $w / d$ values higher than unity, threshold variations become 

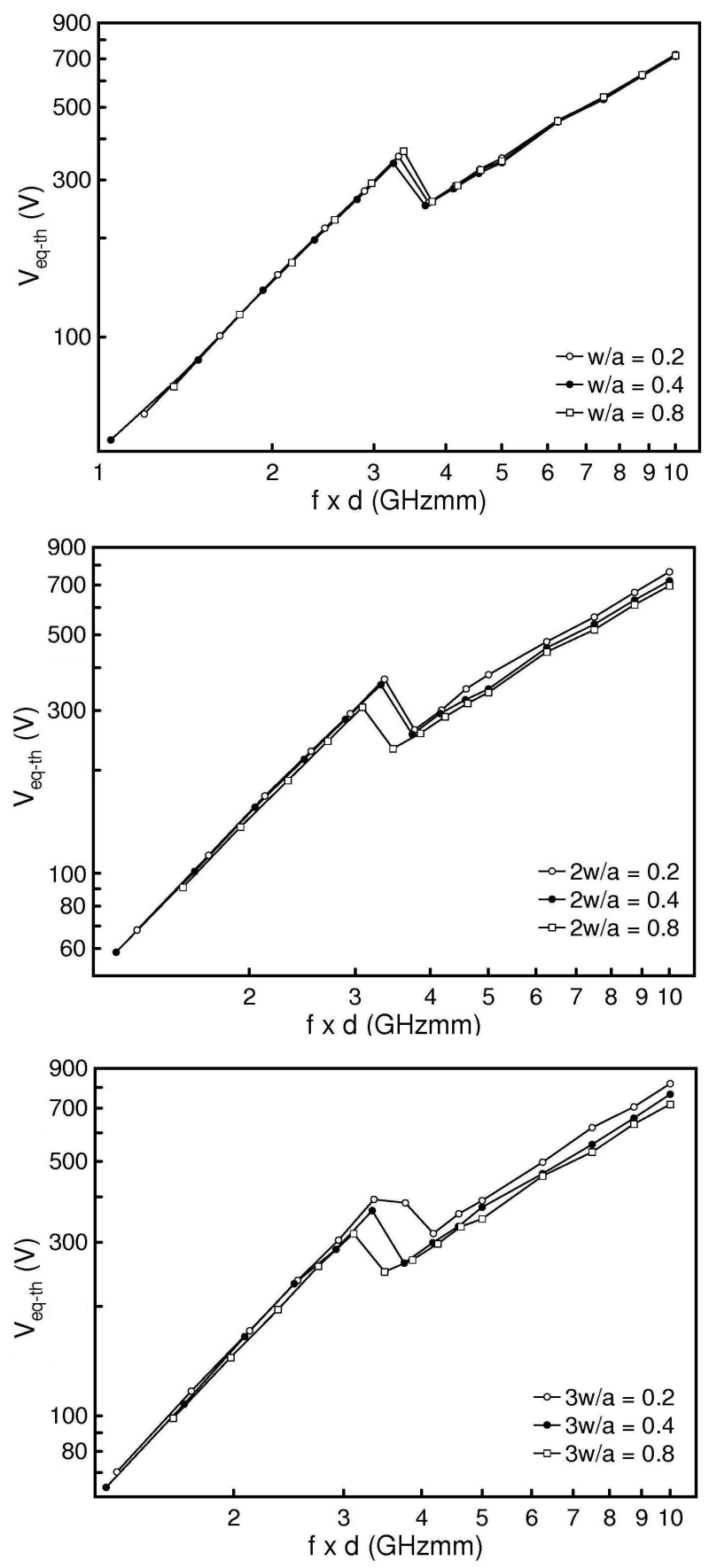

Fig. 6. Multipactor voltage threshold for several ridge widths in asymmetrical configurations. From top to bottom: ridge, double-ridge and multi-ridge waveguides. Gap length is $d=0.5 \mathrm{~mm}, d / b=0.052$ and $b / a=2$.

slighter.

Next, the effect of ridge gap variation on voltage threshold was also studied for both symmetrical and asymmetrical topologies (see Figs. 8 and 9, respectively). Like in the previous performed studies, it is observed a very slight variation of the voltage threshold in terms of the gap for higher $w / d$ values considered.

Finally, multipactor voltage threshold comparison among single, double and multi-ridge waveguide is presented for the symmetrical case in Fig. 10. As it was pointed out in Figs. 5

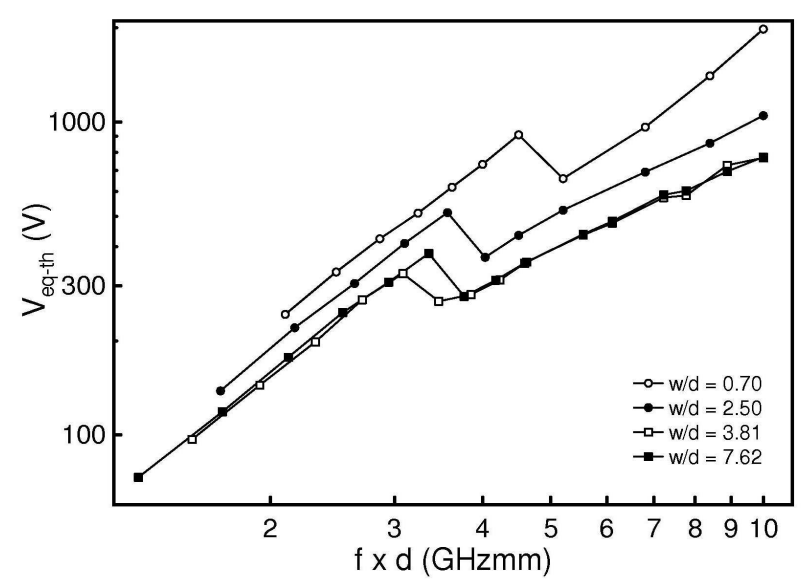

Fig. 7. Multipactor voltage threshold for several values of ridge width to gap ratio for single ridge symmetrical waveguide. Gap length is $d=0.5 \mathrm{~mm}$, $d / b=0.052$ and $b / a=2$.

and $6, \mathrm{RF}$ voltage threshold increases as the ridge width becomes smaller. Multi-ridge waveguide has the narrowest ridges and therefore the higher threshold values, as it can be expected.

\section{Multipactor PRediction using Susceptibility CHARTS}

In this section, a simple multipactor prediction method for complex microwave devices containing ridge waveguide sections is presented. This procedure does not require any additional multipactor simulation. Only the electromagnetic field distribution inside the structure at the operating frequency is needed. Once the electromagnetic fields are computed, the electric field must be integrated in order to calculate the voltage across the ridge gap sections using (1). As the electric field typically varies with the axial direction [27], such voltage must be evaluated for several axial points along the ridge length. As a result, the highest voltage $V_{e q}^{\prime}$ at each ridge in the structure for an RF power input excitation, i.e. $P_{i n}=1 \mathrm{~W}$, is found. For each frequency value, the device input power is proportional to the square of the ridge voltage. Accordingly, the multipactor input power threshold $P_{t h}$ at the input port of the device related to a particular ridge section will be

$$
P_{t h}=\left|\frac{V_{e q-t h}}{V_{e q}^{\prime}}\right|^{2} P_{i n}
$$

where the voltage threshold $V_{e q-t h}$ depends on the frequency gap $f \times d$ of each particular section, which can be extracted from the previously computed multipactor susceptibility charts. Multipactor power threshold in the full structure is the lowest power value obtained among all studied ridges. We detail this method through the two following examples.

\section{A. Evanescent mode filter}

The first analyzed structure is an evanescent mode filter extracted from [28]. Fig. 11 shows the filter topology, and their dimensions are summarized in Table I. Input and output 


\section{Page 5 of 8}
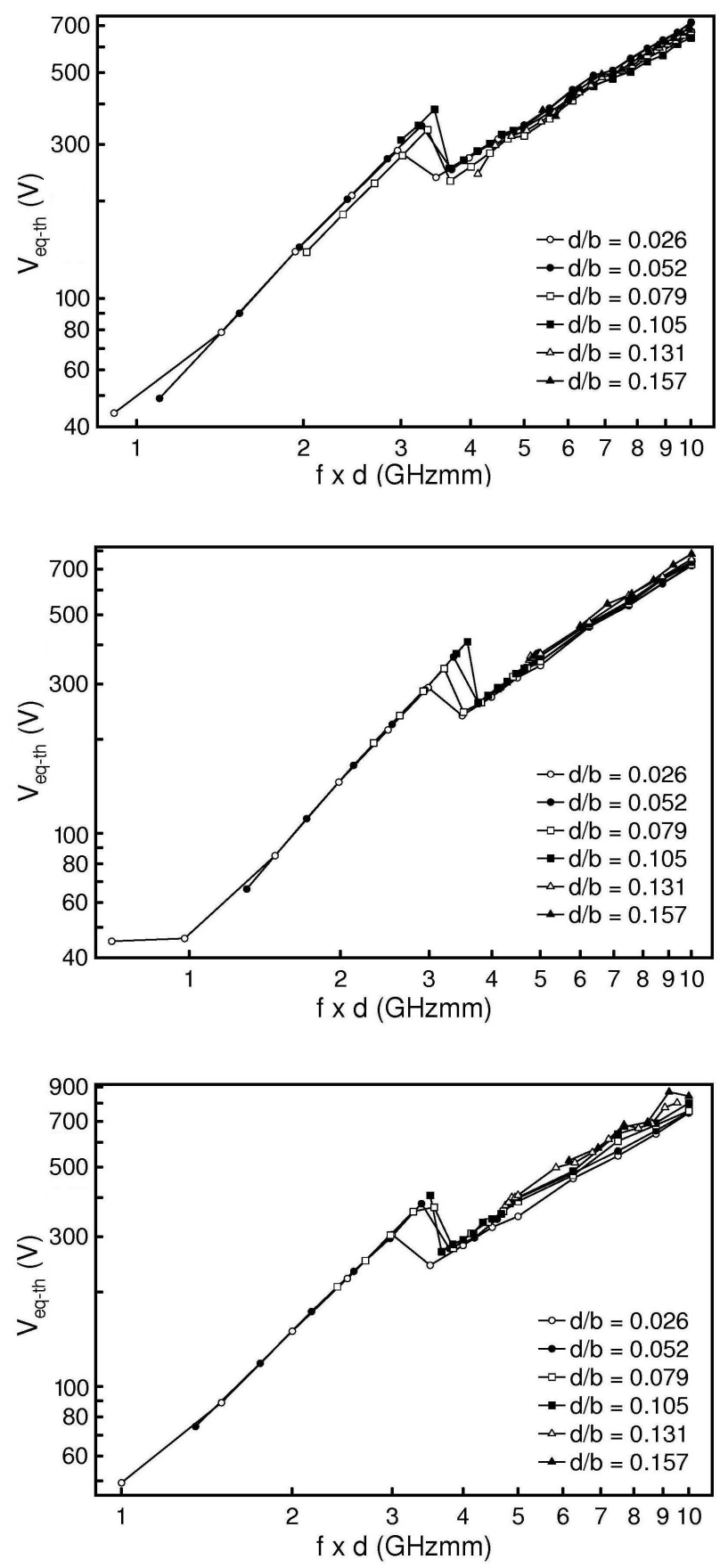

Fig. 8. Multipactor voltage threshold for several gap lengths in symmetrical topologies. From up to down: ridge (width $w / a=0.4$ ), double-ridge $(2 w / a=0.4)$ and multi-ridge $(3 w / a=0.4)$ waveguides. In all cases, $b / a=2$.

ports are implemented with WR137 waveguide $\left(a_{i n}=34.85\right.$ $\mathrm{mm}, b_{\text {in }}=15.80 \mathrm{~mm}$ ).

The operation frequency is chosen to be centered in the passband of the filter, namely $f=9.78 \mathrm{GHz}$. Since the considered structure consists of five identical ridges, the frequency gap product is $f \times d=3.08 \mathrm{GHzmm}$ for all of them. First, electromagnetic analysis of the full structure is performed, assuming $P_{i n}=1 \mathrm{~W}$ excitation at the input port. The equivalent voltage over the gap has been computed for
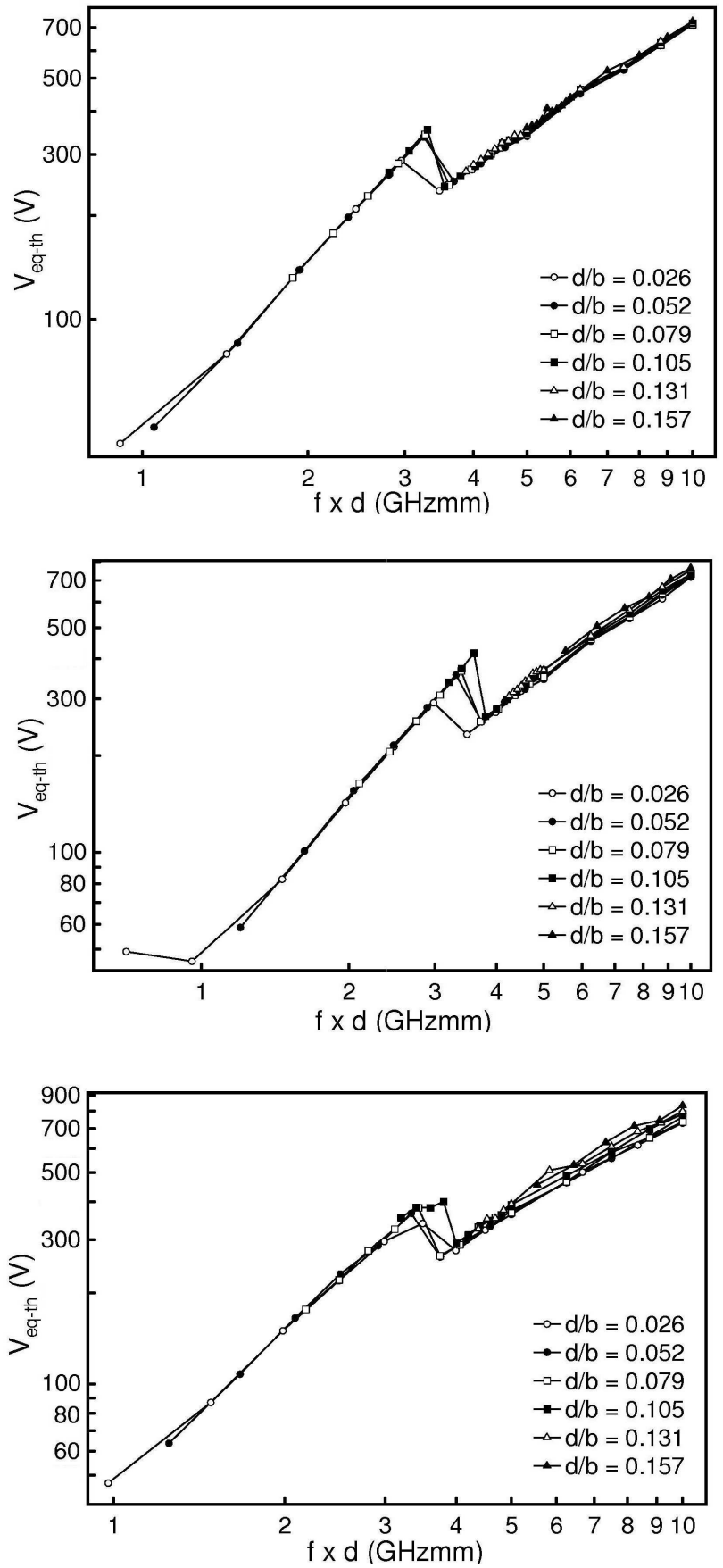

Fig. 9. Multipactor voltage threshold for several gap lengths in asymmetrical topologies. From up to down: ridge (width $w / a=0.4$ ), double-ridge $(2 w / a=0.4)$ and multi-ridge $(3 w / a=0.4)$ waveguides. In all cases, $b / a=2$.

several axial points along the ridge sections (see Fig. 12). Ridges are numbered from the input port to the output port as indicated in Table I. From these results, multipactor threshold can be predicted as follows. Since the ridge sections have the very same gap values, the voltage threshold $V_{e q-t h}$ will be the same for all of them. Therefore, the greatest voltage among all the ridge sections must be found. From such a voltage value and the voltage threshold extracted from symmetrical waveguide charts, multipactor power threshold 


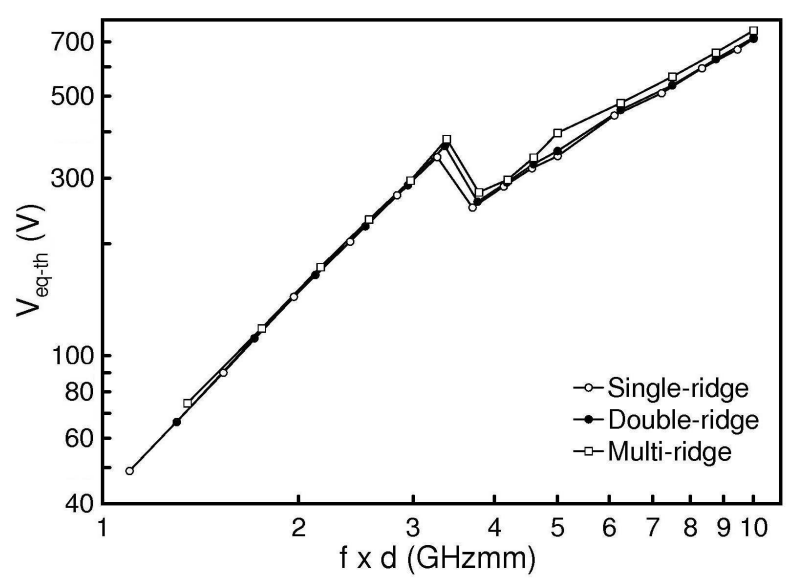

Fig. 10. Multipactor voltage threshold for symmetrical ridged waveguides. Singe-ridge $w / a=0.4$, double-ridge $2 w / a=0.4$ and multi-ridge $3 w / a=$ 0.4 . Gap is $0.5 \mathrm{~mm}(d / b=0.052)$ and $b / a=2$.

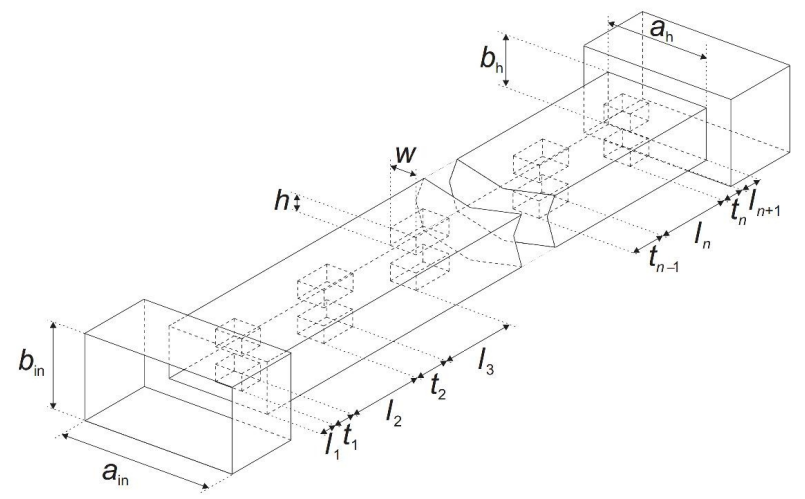

Fig. 11. Symmetrical evanescent mode ridge waveguide filter under consideration.

at the input port can be obtained. Ridge sections have the following dimensions: $w / a=0.45$ and $d=0.315 \mathrm{~mm}$. The most similar situation in previously computed susceptibility charts is found in Fig. 8 for $w / a=0.4$ and $d / b=0.026$. From there, voltage threshold for the working frequency is $V_{e q-t h}=310 \mathrm{~V}$. Moreover, multipactor input power threshold is also computed using FEST3D [23]. Results obtained from both methods are summarized in Table II. The first column indicates the number of the ridge section providing the highest

TABLE I

DIMENSIONS OF THE EVANESCENT MODE FILTER SHOWN IN FIG. 11

\begin{tabular}{|c|c|}
\hline$a_{h}$ & $6.790 \mathrm{~mm}$ \\
\hline$b_{h}$ & $5.275 \mathrm{~mm}$ \\
\hline$w$ & $3.050 \mathrm{~mm}$ \\
\hline$h$ & $2.480 \mathrm{~mm}$ \\
\hline$d$ & $0.315 \mathrm{~mm}$ \\
\hline$l_{1}=l_{6}$ & $0.250 \mathrm{~mm}$ \\
\hline$t_{1}=t_{5}$ & $0.901 \mathrm{~mm}$ \\
\hline$l_{2}=l_{5}$ & $7.785 \mathrm{~mm}$ \\
\hline$t_{2}=t_{4}$ & $1.411 \mathrm{~mm}$ \\
\hline$l_{3}=l_{4}$ & $8.278 \mathrm{~mm}$ \\
\hline$t_{3}$ & $1.411 \mathrm{~mm}$ \\
\hline
\end{tabular}

TABLE II

SUMMARIZED MULTIPACTOR RESULTS FOR THE EVANESCENT MODE FILTER

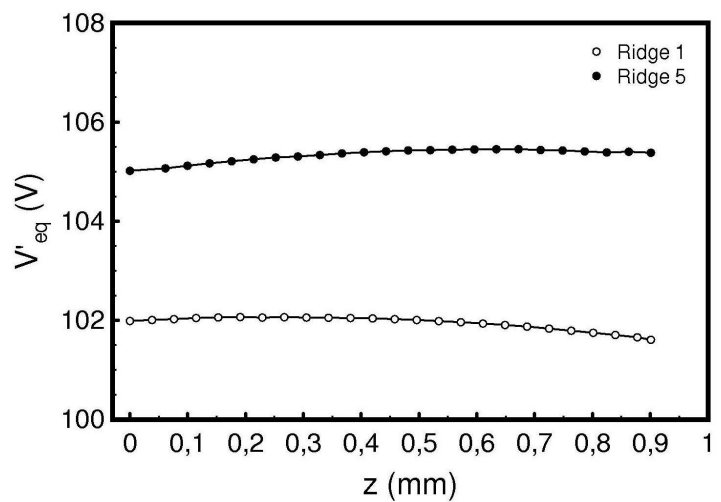

a)

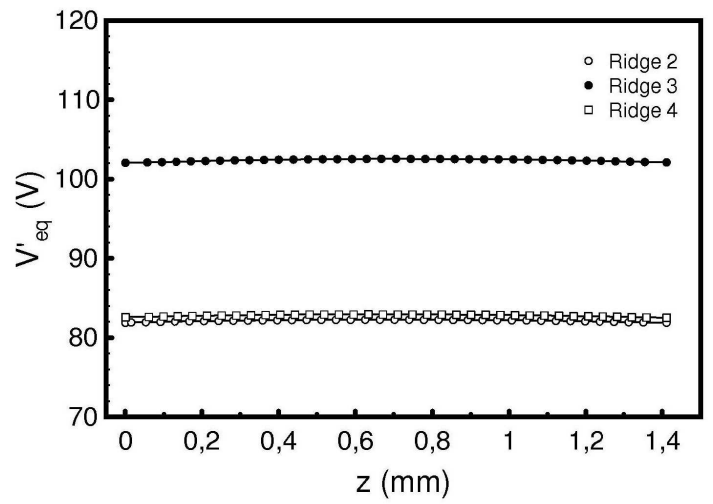

b)

Fig. 12. Equivalent voltage over the gap for $P_{i n}=1 \mathrm{~W}$ on several axial points of the filter. Ridges 1 and 5 in (a), and ridges 2,3 and 4 in (b).

voltage (and consequently where breakdown is expected to occur); the second column gives the highest voltage value for $P_{i n}=1 \mathrm{~W}, V_{e q}^{\prime}$; the third column is the predicted input power threshold, $P_{\text {pred }}$; and the fourth column provides the input power threshold computed by FEST3D, $P_{\text {FEST3D }}$.

It is observed that both simulation and prediction match up with the ridge section where the multipactor appears. In addition, power thresholds obtained by two different procedures are very similar, thus demonstrating the feasibility of the prediction method. Note that the predicted threshold value is always expected to be less than the simulated one, due to the fact that in the susceptibility charts the ridge waveguides have uniform fields along the axial direction. In a realistic case the waveguide is connected to the adjacent discontinuities, and the voltage along the waveguide varies, as depicted in Fig. 12. This effect raises the multipactor threshold above the uniform axial case.

Finally, it must be pointed out the advantage of using the 


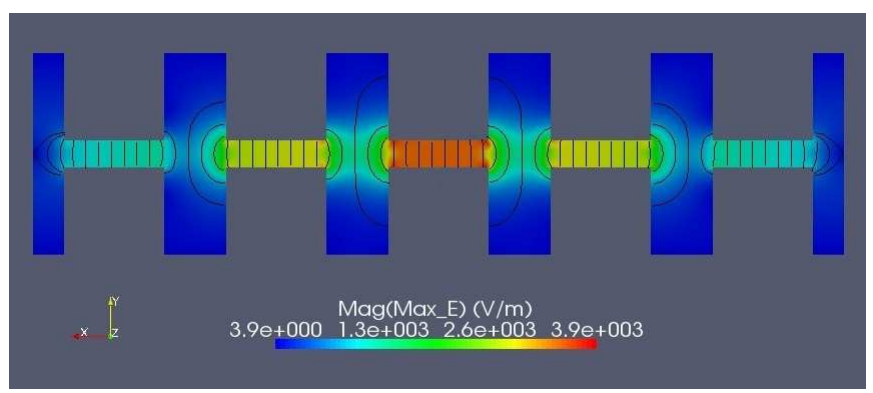

Fig. 13. Electric field lines for multi-ridge waveguide section of the analyzed filter.

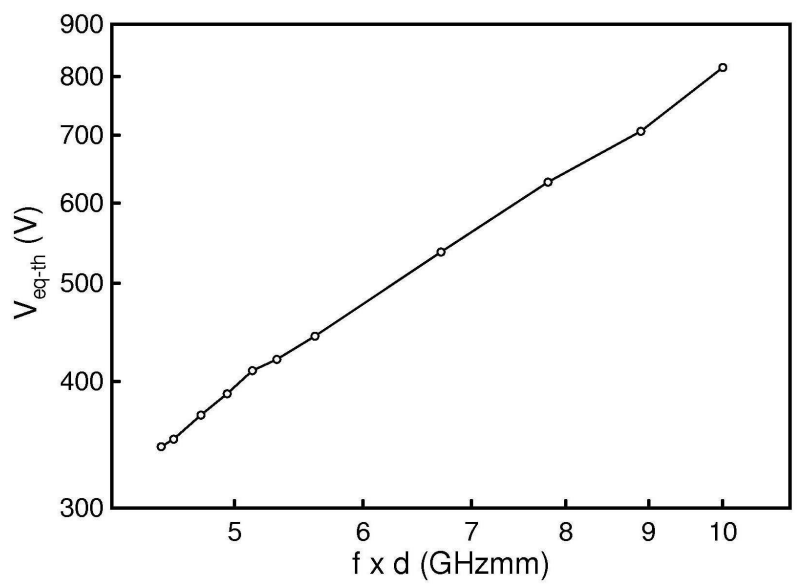

Fig. 14. Multipactor voltage threshold for the symmetrical multi-ridge waveguide of the waffle-iron filter under consideration manufactured in copper.

new computed charts instead of the parallel-plate model charts. From ECSS Multipactor Tool version 1.1 [6], power threshold for the considered gap at the working frequency is $3.44 \mathrm{~W}$ $(192.5 \mathrm{~V})$. Comparing this value with the predicted one using the new generated charts, it is found that the free-multipactor power handling capability of the filter can be increased in 4 dB. In fact, it is well known that parallel-plate model is rather conservative for more complex waveguide geometries.

\section{B. High-Power S-Band Filter}

The last analyzed structure is a high-power S-band filter whose topology and dimensions are detailed in Fig. 2 of [29]. The filter consists of 7 equal multi-ridge sections, each multiridge transversal cross-section has 5 symmetrical ridges with $d=2.413 \mathrm{~mm}$ gap. In Fig. 13 the electric field lines of the transversal cross-section of the filter, which was constructed in copper, are shown.

First, multipactor susceptibility charts are computed for a single 5-symmetrical multi-ridge waveguide with transversal cross-section dimensions of the filter. Results for copper are shown in Fig. 14. For the copper SEY simulations we have used: $W_{1}=35 \mathrm{eV}, \delta_{\max }=2.3$, and $W_{\max }=165 \mathrm{eV}$.

After that, an electromagnetic analysis of the whole device is performed. Input and output ports are implemented with rectangular waveguides of the following dimensions: $a=72.1360 \mathrm{~mm}, b=6.4516 \mathrm{~mm}$. The operation frequency
TABLE III

SUMMARIZED MULTIPACTOR RESULTS FOR THE WAFFLE-IRON FILTER

\begin{tabular}{|c|c|c|c|}
\hline Ridge & $V_{e q}^{\prime}(\mathrm{V})$ & $P_{\text {pred }}(\mathrm{W})$ & $P_{\text {FEST3D }}(\mathrm{W})$ \\
\hline 7 & 13.921 & 1449 & 1532 \\
\hline
\end{tabular}

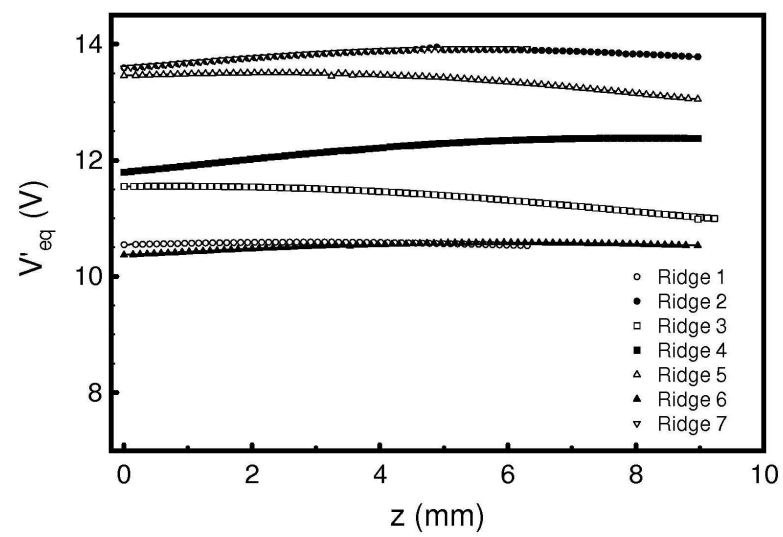

Fig. 15. Equivalent voltage over the gap of the filter ridges for $P_{i n}=1 \mathrm{~W}$ on several axial points.

has been chosen to be $2.78 \mathrm{GHz}$ and the frequency gap product is $f \times d=6.71 \mathrm{GHzmm}$ for all the ridge sections. Equivalent voltage between ridges was calculated for the central ridge (where the electric field is higher), according to Fig. 15. From susceptibility chart in Fig. 14, voltage threshold for the working frequency is $V_{e q-t h}=530 \mathrm{~V}$. With these data multipactor power threshold prediction can be done. Table III summarizes the main results $\left(V_{e q}^{\prime}, P_{\text {pred }}, P_{\text {FEST3D }}\right.$ already defined in the previous subsection).

Good agreement has been found between our predicted value and the one provided by FEST3D. Comparing the predicted power threshold value with the one corresponding to the parallel plate model $(676 \mathrm{~W})$, there is relevant difference of $3.3 \mathrm{~dB}$ between them.

\section{Conclusions}

In this letter, we have studied the multipactor effect in ridged waveguides. Multipactor susceptibility charts have been computed by means of the commercial software FEST3D for several ridge and multi-ridge configurations. For each configuration, variation of the voltage threshold as a function of the different waveguide dimensions is presented and a qualitative explanation of such behaviors in terms of the fringing effect is outlined. Afterwards, developed multipactor susceptibility charts have been used to predict the RF input power threshold for an evanescent mode filter and for a high-power S-band filter, both containing ridges. Predicted multipactor values have been compared with FEST3D simulations of the entire structures and a very good agreement has been found in both cases.

\section{REFERENCES}

[1] J. Vaughan, "Multipactor", IEEE Trans. Electron Devices, vol. 75, no. 6, pp. 1218-1221, August 1995. 
[2] F. Zimmermann, "A simulation study of electron cloud instability and beam induced multipacting in the LHC, CERN, Geneva, Switzerland, CERN-LHC-Project-Report-95, February 1997.

[3] M. A. Furman, "The Electron-Cloud Eect in the Arcs of the LHC, CERN, Geneva, Switzerland, CERN-LHC-Project-Report-180, May 1998.

[4] J.R.M. Vaughan "Secondary Emission Formulas", IEEE Trans. Electron Devices, vol. 40, no. 4, p. 830, April 1993.

[5] A. Woode, J.Petit, "Diagnostic investigations into the multipactor effect, susceptibility zone measurements and parameters affecting a discharge, Technical Report, ESA/ESTEC Working Paper no. 1556, Noordwijk (The Netherlands), November 1989.

[6] "Space Engineering: Multipacting Design and Test", ESA Publication Division, The Netherlands, ECSS-20-01A, edited by ESA-ESTEC, May, 2003.

[7] A. J. Hatch, H. B. Williams, "Multipactor Modes of High-Frequency Gaseous Brekdown", The Physical Review, Second Series, vol. 112, no 3, pp. 681-685, November 1958.

[8] J. Lara, F. Pérez, M. Alfonseca, L. Galán, I. Montero, E. Román, D. Raboso, "Multipactor prediction for on-board spacecraft RF equipment with the MEST software tool, IEEE Transactions on Plasma Science, vol. 34, no. 2, pp. 476-484, April 2006.

[9] R. A. Kishek, Y. Y. Lau, L. K. Ang, A. Valfells, R. M. Gilgenbach, "Multipactor discharge on metals and dielectrics: Historical review and recent theories", Physics of Plasmas, vol. 5, no. 5, pp. 2120-2126, May 1998.

[10] A. M. Pérez, C. Tienda, C. Vicente, S. Anza, J. Gil, B. Gimeno, V. E. Boria, D. Raboso, "Prediction of multipactor breakdown thresholds in coaxial transmission lines for traveling, standing, and mixed waves", IEEE Transactions on Plasma Science, vol. 37, no. 10, pp. 2031-2040, October 2009.

[11] R. Udiljak, D. Anderson, M. Lisak, V. Semenov, J. Puech, "Multipactor in a coaxial transmission line. Part I: Analytical study", Physics of Plasmas, vol. 14, 033508, 2007

[12] V. Semenov, N. Zharova, R. Udiljak, D. Anderson, M. Lisak, J. Puech, "Multipactor in a coaxial transmission line. Part II: Particle-in-cell simulations", Physics of Plasmas, vol. 14, 033509, 2007.

[13] E. Somersalo, P. Y1-Oijala, D. Proch, "Electron multipacting in RF structures, Deutsches Elektronen-Synchrotron DESY, Hamburg, Germany, TESLA Rep. 94-14, July 1994.

[14] C. Vicente, M. Mattes, D. Wolk, B. Mottet, H.L. Hartnagel, J.R. Mosig and D. Raboso, "Multipactor breakdown prediction in rectangular waveguide based components", Microwave Symposium Digest, 2005 IEEE MTT-S International, 12-17 June 2005.

[15] V. E. Semenov, E. I. Rakova, D. Anderson, M. Lisak, J. Puech, "Multipactor in rectangular waveguides", Physics of Plasmas, vol. 14, 033501, 2007.

[16] V. E. Semenov, E. I. Rakova, A. G. Sazontov, I. M. Nefedov, V. I. Pozdnyakova, I. A. Shereshevskii, D. Anderson, M. Lisak, J. Puech, "Simulations of multipactor thresholds in shielded microstrip lines", Journal of Physiscs D: Applied Physics vol. 42, 205204, 2009

[17] V. E. Semenov, N. A. Zharova, D. Anderson, M. Lisak, J. Puech, "Simulations of multipactor in circular waveguides", Physics of Plasmas, vol. 17, 123503, 2010.

[18] A. M. Pérez, V. E. Boria, B. Gimeno, S. Anza, C. Vicente, J. Gil "Multipactor analysis in circular wave-guides", Journal of Electromagnetic Waves and Applications vol. 23, pp. 1575-1583, 2009.

[19] A. Frotanpour, G. Dadashzadeh, M. Shahabadi, B. Gimeno, "Analysis of Multipactor RF Breakdown Thresholds in Elliptical Waveguides", IEEE Transactions on Electron Devices vol. 58, no. 3, pp. 876-881, March 2011.

[20] J. Uher, J. Bornemann, U. Rosenberg, "Waveguide Components for Antenna Feed System: Theory and CAD”, Norwood, MA: Artech House 1993.

[21] FEST3D, ESA/ESTEC, Valencia, Spain, [Online]. Available: www.fest3d.com

[22] G. Conciauro, M. Guglielmi, and R. Sorrentino, Advanced Modal Analysis, New York, NY: John Wiley \& Sons Inc, 2000.

[23] S. Anza, C. Vicente, D. Raboso, J. Gil, B. Gimeno and V. E. Boria, "Enhanced prediction of multipactor breakdown in passive waveguide components including space charge effects", Proc. IEEE MTT-S Microw. Symp. Dig., 2008, pp. 1095-1098.

[24] D. Wolk, C. Vicente, H.L. Hartnagel, M. Mattes, J.R. Mosig, D. Raboso "An investigation on the effect of fringing fields on multipactor breakdown", 5th International Workshop on Multipactor, Corona and Passive Intermodulation, September 2005.
[25] R. Udiljak, D. Anderson, M. Lisak, J. Puech, and V.E. Semenov, "Multipactor in a Waveguide Iris", IEEE Transactions on Plasma Science, vol. 35, no. 2, pp. 388-395, April 2007.

[26] V. E. Semenov, E. Rakova, R. Udiljak, D. Anderson, M. Lisak, and J. Puech, "Conformal mapping analysis of multipactor breakdown in waveguide irises", Physics of Plasmas, vol. 15, 033501, 2008.

[27] P. Sarasa, A. González, H. Esteban, P. Mader, K. Tossou, P. Lepeltier, "Comparative Study of the Power Handling Capability of Space Broadband Antenna Filters in Ku-Band", 5th International Workshop on Multipactor, Corona and Passive Intermodulation, September 2005.

[28] P. Soto, D. de Llanos, V. E. Boria, E. Tarín, B. Gimeno, A. Oñoro, I. Hidalgo, and M. J. Padilla, "Performance analysis and comparison of symmetrical and asymmetrical configurations of evanescent mode ridge waveguide filters", Radio Science, vol. 44, RS6010, doi:10.1029/2008RS004034, 2009.

[29] H. Guthart, "A High-Power S-Band Filter", IRE Transactions on Microwave Theory and Techniques, vol. 10, pp. 148-149, 1962. 